\section{CHOLECYSTECTOMY WITHOUT DRAINAGE *}

\section{A. MURAT WILLIS, M.D.}

Fellow of the American College of Surgeons; Professor of Clinical and Operative Surgery, Medical College of Virginia RICHMOND, VA.

At this time gallbladder surgery is occupying the attention of surgeons perhaps more than any other subject. The reason for its prominence is obvious. Cholecystostomy, once the operation of choice, has been denounced by an able coterie of surgeons as giving permanent results in not more than half of the cases; and cholecystectomy, a supposedly more dangerous operation, is being substituted for it whenever conditions allow.

All are familiar with the condition found at operation after a previous cholecystostomy. Often the liver is turned up at its lower border and lies on a level with or above the costal margin, having diaphragmatic and peritoneal adhesions. The duodenum, transverse colon and stomach are often adherent to the gallbladder and the liver. I believe this is due entirely to the drainage, which does not allow the liver to assume its normal position, and which, in my opinion, is responsible for the adhesions to the other structures. In

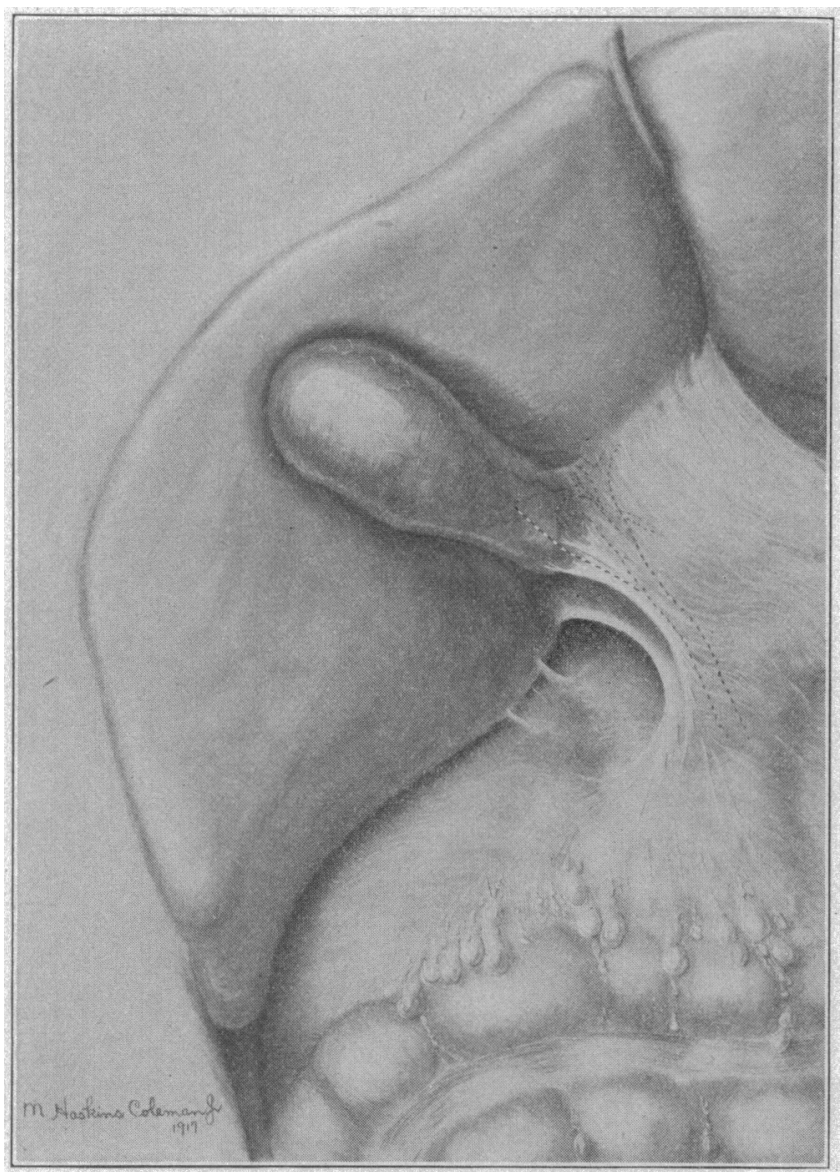

Fig. 1.-Relations of cystic duct to common duct. Dotted lines indicate direction of incision in hepatoduodenal ligament.

every case in which I have done a cholecystostomy and later gone back into the abdominal cavity, I have found a mass of adhesions about the gallbladder.

- Read before the Maryland Medical and Chirurgical Society, Baltimore, April 25, 1917.
In fact, I have never seen, in secondary operations elsewhere in the abdomen, the same number and density of adhesions. I have never found a case which did not have this condition. It is impossible for me to state from my operative observation how

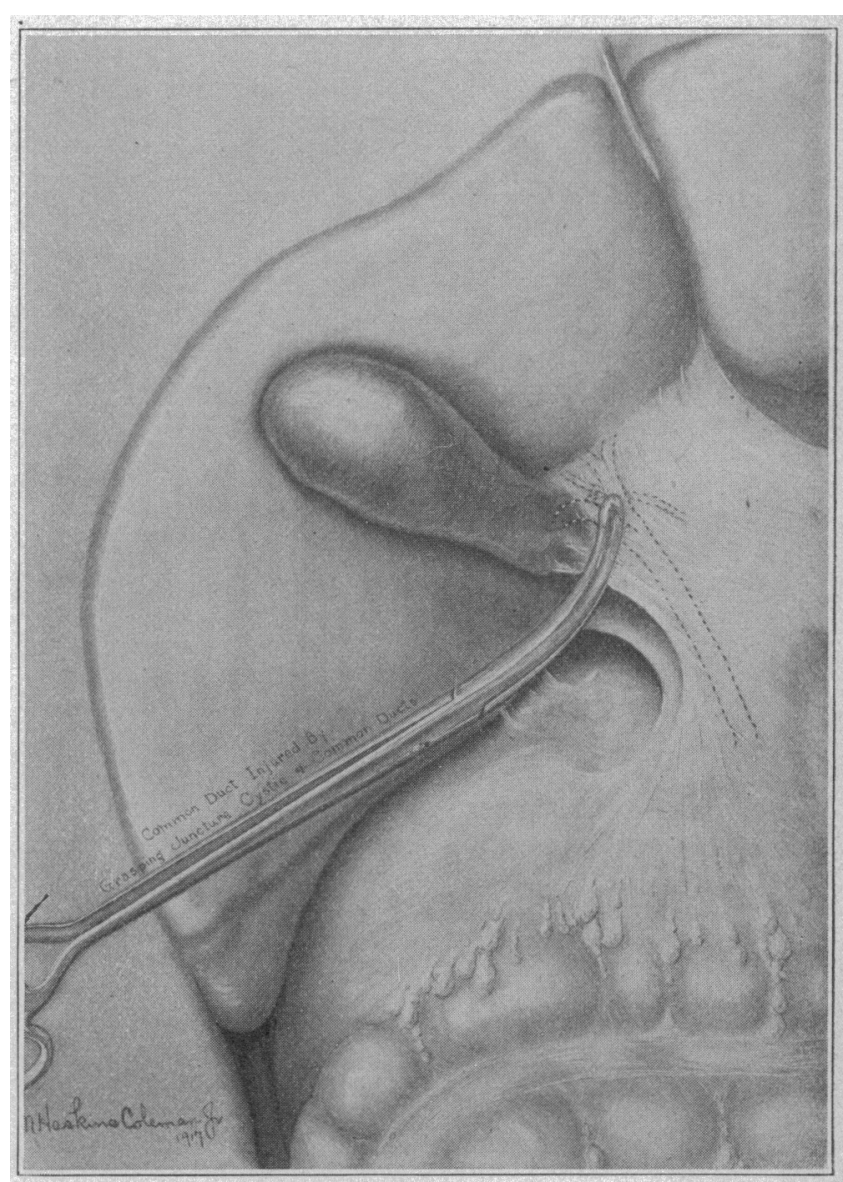

Fig. 2.-Method generally employed. When making sure of the cystic duct, the curved artery clamp is apt to grasp and injure the com mon duct as well.

many adhesions form about the gallbladder region after a cholecystectomy without drainage, as I have never had occasion to reopen the abdomen in such cases. I feel confident that fewer adhesions would be found associated with a more normal condition of this region. I am sure, however, that this result will be obtained only with careful removal, allowing no bile to escape into the cavity.

In some recent experiments by Dr. Budd and myself, we were able to demonstrate that presumably healthy bile, if spilled in the region of the gallbladder, would produce a moderate amount of adhesions, and that bile infected with the colon and typhoid bacillus would produce extensive adhesions. We were also able to show that infected bile spilled in the region of the gallbladder, plus drainage, were followed by even more extensive adhesions. We removed gallbladders without liberating bile in the cavity, closing without drainage, and in these cases the number of adhesions was negligible.

About five years ago, we were first impressed with the number of cases of cholecystectomy which never leaked any bile through the drain after operation. We began, in carefully selected cases, to close without drainage, and found that excellent results followed. We began to enlarge more and more on this group, 
until now we remove quite a large percentage of gallbladders without drainage. We do not believe that all cases can be closed without a drain, but do contend that a big percentage can be done in this way, provided,

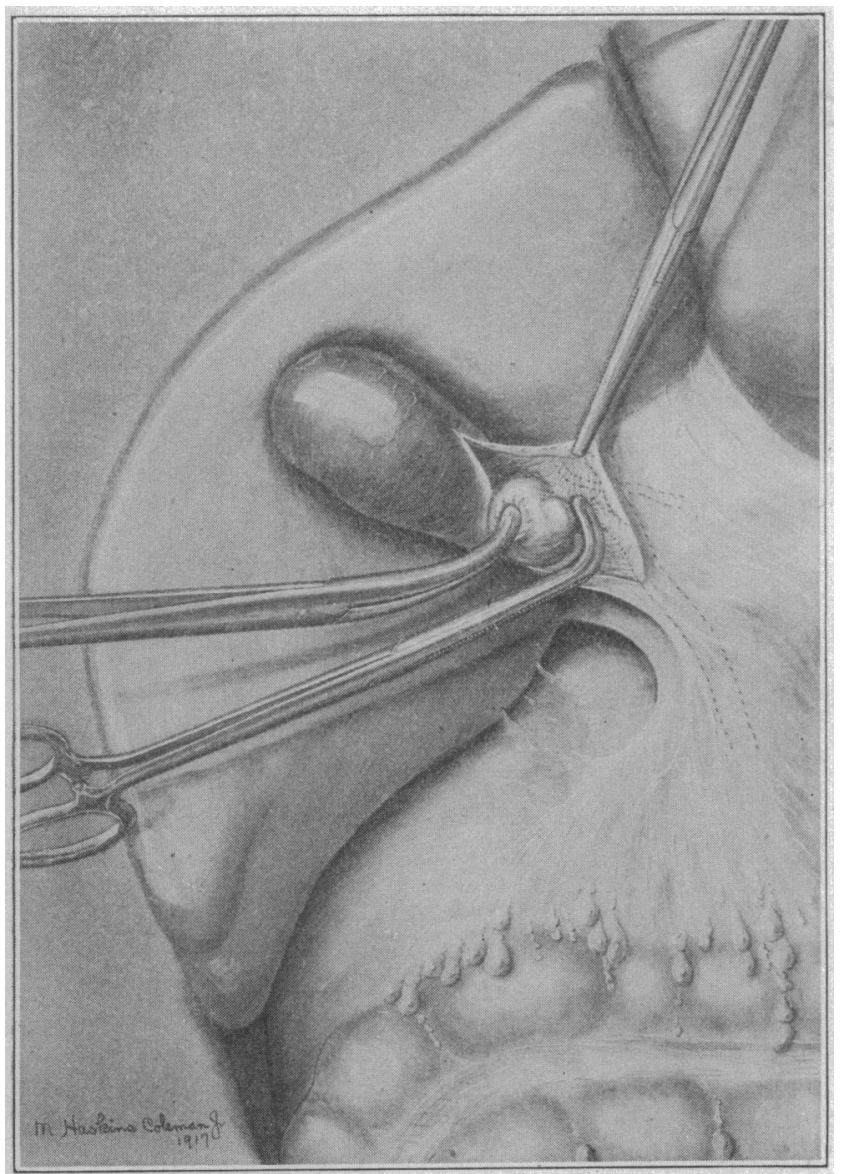

Fig. 3.-Incision made in hepatoduodenal ligament. Pouch of gallbladder rotated up, and right angle clamp placed on cystic duct and artery.

of course, the surgeon is careful to spill no bile in the cavity and to leave no bleeding surfaces.

We believe that the technic of the removal of the gallbladder, whether from the fundus down or from below upward, is largely a matter of each surgeon's preference as to the method by which he can best control the situation. I myself believe that the removal from below toward the fundus offers the greatest advantages.

\section{OPERATIVE TECHNIC}

Using the right rectus incision curving at the upper end toward the xiphoid, we bring the gallbladder at once into view. Rotating the liver upward, we make an incision in the hepatoduodenal ligament, as shown by the dotted line in Figure 1, and by blunt dissection bring the cystic duct into the field.

The method generally employed is to place a curved clamp over this ligament, as shown in Figure 2. We believe that this in itself is responsible for many reported injuries to the common duct in cholecystectomy, as the clamp, in being placed sufficiently far to assure the grasping of cystic duct and artery, is liable to grip the common duct as well. There is also a possibility of a ligature being put through or around the common duct when the cystic duct is tied off, or of the scissors injuring it when the cystic duct is cut. It is to be borne in mind that the cystic duct does not join the common duct at right angles in a great many instances, but runs parallel to it and right alongside.

We believe that this accident is obviated absolutely in our method. Grasping the pelvis of the gallbladder and pulling upward, we are able to apply a right angle clamp on the cystic duct and artery, as shown in Figure 3. Figure 4 illustrates what we have found to be the usual relations of the cystic duct to the pelvis of the gallbladder, certainly in pathologic conditions. Then, a No. 2 catgut ligature is placed around the cystic duct close up to the junction with the common duct, and we are careful to cause no stricture of the latter. We thus avoid leaving sufficient cystic duct to form a bud in which future trouble is liable to occur. Another ligature is now placed around the cystic duct and artery, and the two are cut (Fig. 5). By blunt dissection with the back of the knife and the finger, the gallbladder is dissected back to the fun-

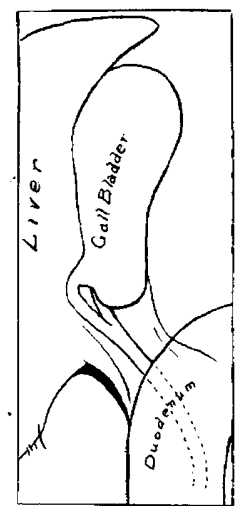

Fig. 4. - Sem: schem a tic view illustrating th e usual relations of cystic duct to pelv
of gallbladder. dus, care being taken to leave the peritoneal coat of the liver intact. The gallbladder is removed, sufficient peritoneum being allowed to remain to close the space

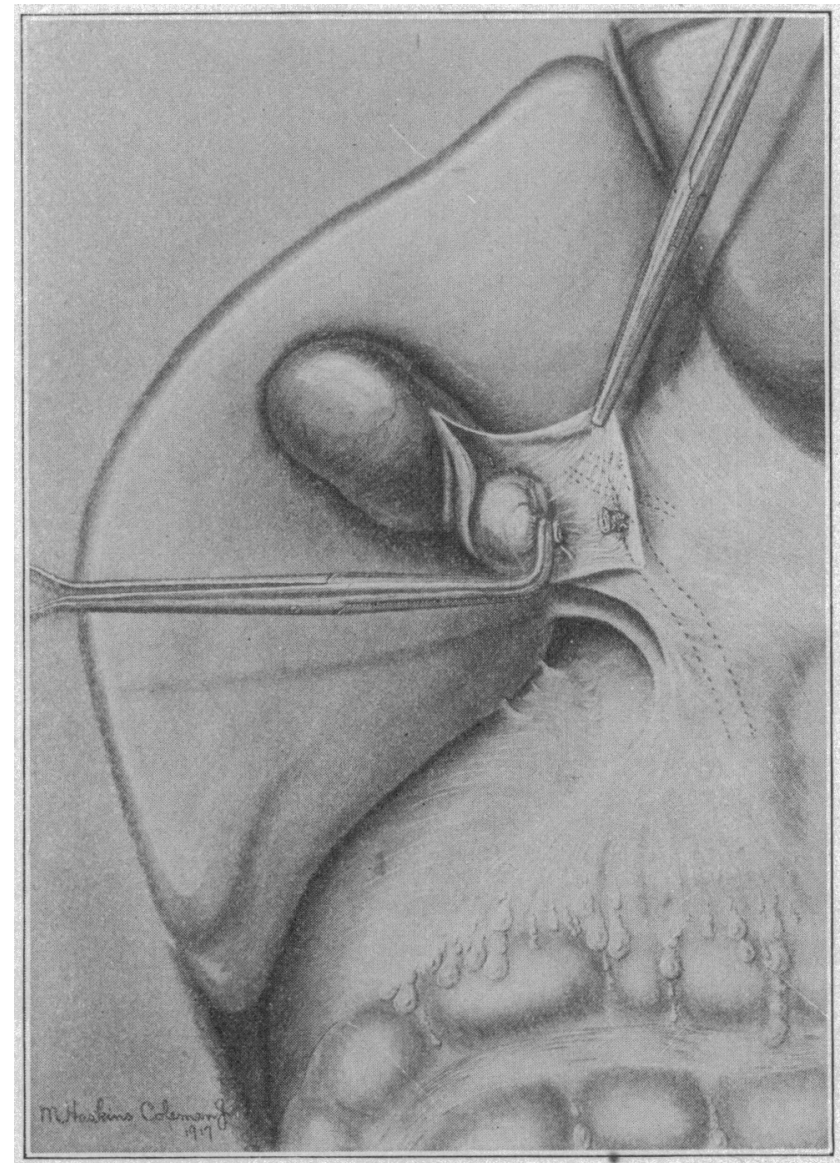

Fig. 5.-Ligature placed around cystic duct, also around cystic duct and artery close to common duct, and cut.

without tension. A crown suture is placed through the peritoneum and under the stump of the cystic duct, being brought back over the stump and tied, as shown in Figure 6. This drops the stump back in the liga- 
ment, and a continuous suture closes all raw surfaces and leaves a clean field, as shown in Figure 7 . We then close without drainage, and have no fear that bile will escape into the peritoneal cavity.

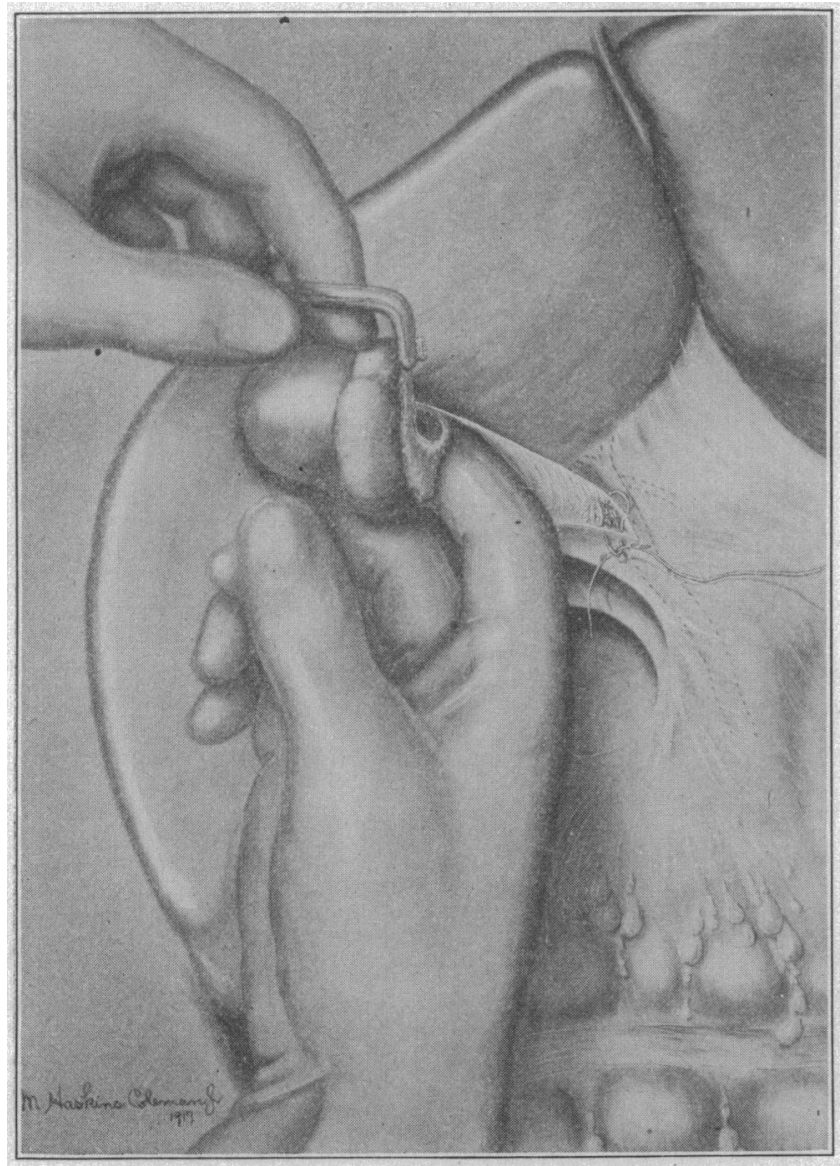

Fig. 6.-. Crown suture in place under and over cystic stump. Gallbladder being dissected back to fundus.

\section{RESULTS OF OPERATIONS}

Our experience in surgery of the gallbladder is based on 549 operations for cholelithiasis and cholecystitis with twelve deaths, or 2.2 per cent. In this series, 466 patients, or 86 per cent., had gallstones in the bladder or duct; eighty-two patients, or 14 per cent., had no gallstones, but showed various stages of cholecystitis. There were 398 cholecystostomies with seven deaths, or 1.7 per cent., and 107 cholecystectomies with one death, or 0.9 per cent. In thirty-eight of our cholecystectomies, we left no drainage. It was in this group that the one death occurred. There were forty-four choledochotomies with four deaths, practically 9 per cent.

There were twenty-six secondary operations. Twenty-one followed the operation of cholecystostomy and five occurred after choledochotomy. Gallstones were found in twelve of the secondary operations; seven in the cholecystostomy and in all five of the choledochotomy group.

This series presents several interesting facts. The women outnumbered the men five to one. The percentage of deaths in the common duct cases was about five times as great as the mortality in other gallbladder work. The causes of death in the common duct group were secondary hemorrhage and infection. The mortality in our cholecystectomies is much lower than in the cholecystostomies. This is due to the fact that cholecystectomy on the whole was performed in the more favorable type of case.

The patient lost in our cholecystectomy group was a woman aged 66 with a simple case of cholelithiasis. The gallbladder was ruptured during removal. Drainage was not used, as we were able to cover the raw surface and clean out the peritoneal cavity. She had urinary suppression, and died three days after operation. At necropsy a small abscess was found between the liver and transverse colon, and an acute nephritis was revealed. There was no evidence of the escape of bile. It is impossible to say whether she died from acute nephritis or an infection; but in future, under similar circumstances, we shall drain.

It should be borne in mind that this mortality represents the patients who have died in the hospital after operation on the gallbladder. Some of these patients undoubtedly died of causes other than those which can be attributed to the immediate effect of the operation, but, because of the fact that they died in the hospital, we have included them in the gallbladder mortality.

\section{CONCLUSIONS}

1. Cholecystectomy for simple infections of the gallbladder is a most successful operation, especially if this can be done without soiling the peritoneum by the contents of the gallbladder, and closing without drainage. 2. Adhesions of organs which normally

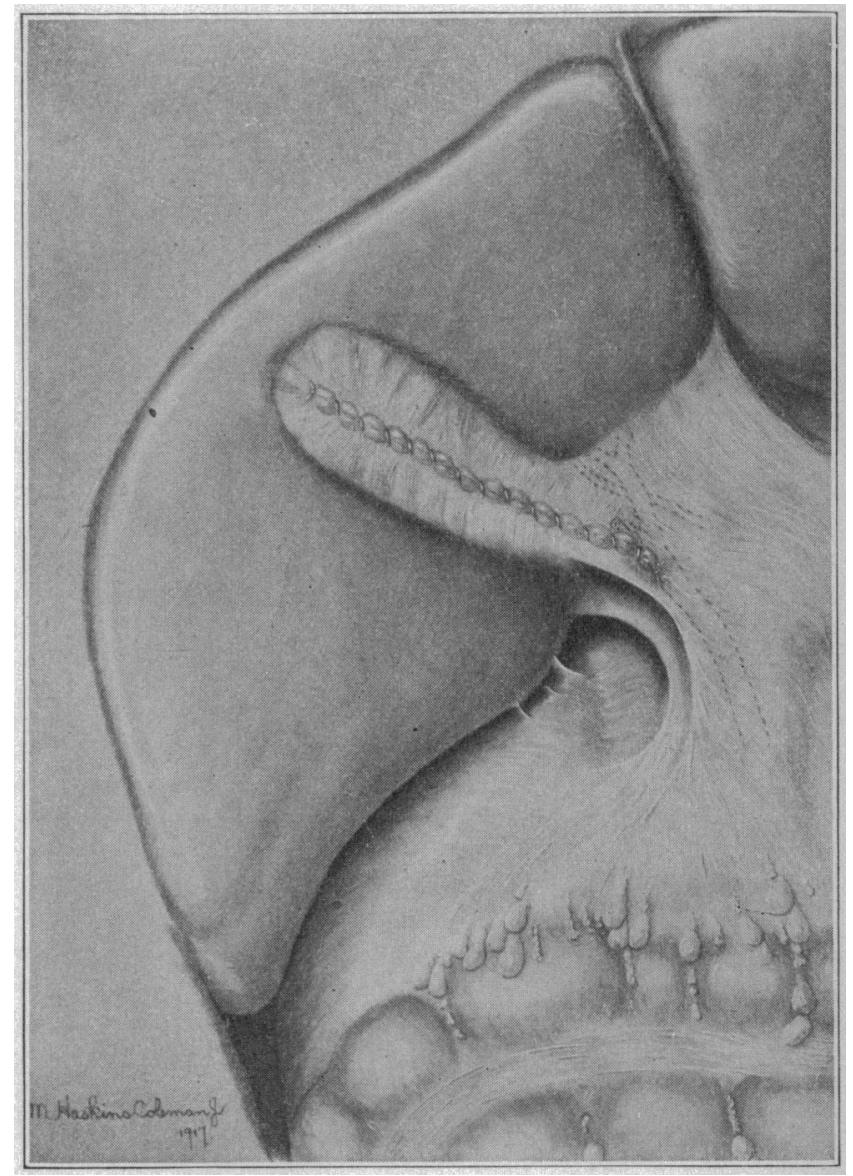

Fig. 7.-All raw surfaces covered, and clear field left ready to close without drainage.

have a certain amount of mobility cause postoperative complaints, especially in the gallbladder region, and we feel that any operation which will limit these is certainly a step in the right direction. 